\title{
Parques tecnológicos y desarrollo regional: una experiencia de Argentina
}

\section{Technology parks and regional development: an experience from Argentina}

\author{
José Ignacio Diez y Yesica Soledad Dilerniab \\ ${ }^{a}$ Universidad Nacional del Sur, Argentina.jdiez@uns.edu.ar \\ ${ }^{b}$ Universidad Nacional del Sur, Argentina.ydilernia@plapiqui.edu.ar
}

\section{RESUMEN}

En las últimas décadas, el mundo viene siendo testigo de importantes cambios tecnoproductivos que están alterando el funcionamiento del sistema capitalista. La economía global se encuentra atravesando una fase producción de carácter postfordista, donde el conocimiento se transforma en un insumo fundamental de la actividad productiva. En este contexto, de primacía de las ideas en las actividades productivas, los territorios buscan diseñar mecanismos para transformarse en verdaderas "usinas de pensamiento aplicado": ya sea para captar capital o bien para generarlo desde cero. Entre los instrumentos de política pública para alcanzar este objetivo, se encuentran los parques tecnológicos, que son iniciativas implementadas por las comunidades para ampliar la base económica de alta tecnología de una localidad o región. El presente trabajo pretende analizar la dinámica de comportamiento de un polo en particular, el Parque Científico Tecnológico del Litoral Centro, situado en la provincia de Santa Fe-Argentina.

Palabras clave: Parques tecnológicos; política pública; desarrollo económico local

\section{ABSTRACT}

In the last decades, the world has been witness to significant technological and productive changes that have altered the dynamics of the capitalist system. The global economy is entering a new production fase called postfordism, in which knowledge has become the most important factor in the productive activity. In this context, in which ideas have an important role in the productive activities, the territories try to build instruments to generate or attract knowledge. Among the instruments to achieve this objective are technology parks. These are iniciatives implemented by local communities in order to extend the economic base of high tech industries in a territory. The present work pretends to analize the dynamics of a particular park, called the litoralcentro technology park which is situated in Santa Fé-Argentina.

Keywords: Technology parks; public policies; local economic development

\section{INTRODUCCIÓN}

En las últimas décadas, el mundo viene siendo testigo de importantes cambios tecno-productivos que están alterando el funcionamiento del sistema capitalista. La aparición del chip y del ordenador como factores claves del proceso de producción, la nueva flexibilidad de los procesos de trabajo y la emergencia de las tecnologías de 
la información y la comunicación, han alterado el patrón de acumulación en la economía global.

De acuerdo a Carlota Pérez (1996), la economía capitalista se encuentra en una fase de producción de carácter postfordista, donde el conocimiento se transforma en un insumo fundamental de la actividad productiva.

Esta nueva forma de producir implica que la fuente fundamental de la productividad de la firma es el acervo de saberes: aquellos de carácter explícito o codificado, que pueden encontrarse en manuales y en el ámbito de la ciencia y de la técnica y los de carácter implícito, es decir aquellos que surgen del propio proceso de trabajo, a través de la acumulación de habilidades.

En este contexto, de primacía de las ideas en las actividades productivas, los territorios buscan diseñar mecanismos para transformarse en verdaderas "usinas de pensamiento aplicado": ya sea para captar capital o bien para generarlo desde cero, a partir de las competencias acumuladas por las empresas e instituciones locales.

Entre los instrumentos más utilizados para alcanzar este objetivo, se encuentran los parques o polos tecnológicos, que son iniciativas implementadas por las comunidades para ampliar la base económica de alta tecnología de una localidad o región.

Pensado como un instrumento de política pública, la conformación de tecnopolos presenta determinadas características que resulta importante remarcar.

En primer término, un parque tecnológico constituye en sí mismo una herramienta de marketing para promocionar la imagen de un territorio y posicionarlo como un ámbito privilegiado para el desarrollo de actividades productivas.

En segundo lugar, un parque tecnológico es una herramienta legal para favorecer una determinada lógica de producción, que supone la articulación públicoprivada (entre empresas y organizaciones científico-tecnológicas), donde es necesario regular las actividades de transferencia y es menester también establecer derechos de propiedad sobre los productos que surgen de estas relaciones.

En tercer término, un polo de estas características también permite la valorización del suelo, generando negocios de naturaleza inmobiliaria, asociados a la ampliación de servicios de infraestructura y a las mejoras edilicias $\mathrm{y}$ medioambientales que se derivan de su generación y puesta en marcha.

En cuarto lugar, el tecnopolo es una forma de polarización existente al interior de un espacio más amplio. En este sentido, constituye una interface entre relaciones productivas basadas en la proximidad y una perspectiva global más amplia que otorga estímulo para un desarrollo dinámico.

En términos estrictamente económicos, un parque de estas características constituye un intento de crear productos de base tecnológica mediante la minimización de los costos de transacción asociados a la colaboración de agentes económicos, que históricamente han contado con obstáculos para vincularse, motivo de restricciones idiosincráticas o institucionalizadas dadas.

Finalmente, constituye también una herramienta para diseminar el progreso técnico en forma transversal sobre todo el tejido económico y social, desarrollando nuevas capacidades tecnoproductivas y fortaleciendo las ya existentes.

El presente trabajo pretende analizar un caso específico de polo, este es el Parque Tecnológico del Litoral Centro (PTLC); el mismo se encuentra radicado en la 
provincia de Santa Fe sobre la ruta Nacional 168 y es uno de los más antiguos de la República Argentina. A lo largo del artículo se apunta conocer su estructura organizativa y su dinámica, con el propósito de entender el papel que el mismo puede tener en el desarrollo económico de la localidad y su zona de influencia.

Específicamente, se pretende dar respuesta a los siguientes interrogantes: ¿Cuáles han sido las causas que dieron origen a este emprendimiento? ¿Cómo se encuentra estructurado? ¿Cuáles han sido sus principales acciones en los últimos años? ¿Qué capacidades ha logrado desarrollar? ¿Cuáles son las principales limitaciones que enfrenta para su crecimiento y consolidación? ¿En qué medida el contexto en el que se desenvuelve el parque puede favorecer su mejor desenvolvimiento o, por el contrario, lo condiciona?

Dado la ausencia de estudios de esta naturaleza en Argentina, lo reciente que son las iniciativas de parques tecnológicos en este país y la ausencia de metodologías específicas para analizarlos, es que el presente trabajo es mayormente exploratorio y de naturaleza descriptiva. En este sentido, se pretende que sirva como base para el desarrollo de futuras investigaciones en este campo.

\section{ORgANIZACIÓN DE LA INVESTIGACIÓN}

El artículo se encuentra dividido en seis secciones. En la primera de ellas, se trabaja la idea o noción de Parque Tecnológico. Como puede observarse a lo largo de la misma, no existe una definición unívoca del término, ya que se trata de un concepto que presenta cambios de interpretación según los tiempos, las condiciones culturales, políticas y socioeconómicas de los países.

En segundo lugar, se realiza una breve descripción del origen de los Parques Tecnológicos y sus principales características. Aquí se sintetizan en forma breve diferentes experiencias de distintas partes del mundo, específicamente de América del Norte, Europa, Asia, y América Latina. Aquí se muestra que las experiencias que se registran a nivel mundial son sumamente heterogéneas, evidenciándose diferencias en la estructura organizativa de los distintos polos y los sectores que se promueven, entre otras cuestiones.

En tercer término, se analiza el caso particular del Parque Tecnológico del Litoral Centro. En esta sección se resaltan sus rasgos fundamentales, la dinámica de los actores que intervienen en él y su forma de funcionamiento, a luz de lo sostenido por la teoría. En este apartado también se describe la historia de su conformación, su forma organizativa y se ilustra con algunos números el impacto que la institución tiene sobre la economía local y regional.

En cuarto lugar, se genera un análisis FODA para determinar las principales fortalezas y debilidades que presenta el PTLC como instrumento de política pública. A su vez, también se analizan las perspectivas que ofrece el entorno para el desenvolvimiento de la organización. A partir de entrevistas a las autoridades del mismo, encuestas realizadas a los empresarios ${ }^{1}$ y documentos, se logran identificar estos elementos, que otorgan una verdadera dimensión de aquellas virtudes y restricciones vigentes en el accionar cotidiano de la entidad.

Finalmente, se presentan las conclusiones de la investigación. En esta sección se recogen y sistematizan las evidencias y opiniones expresadas en los apartados

\footnotetext{
${ }^{1}$ Las encuestas a empresarios se realizaron a través de la plataforma online e-encuestas.com.
} 
anteriores, de forma tal de brindar un panorama general respecto a los principales desafíos que enfrenta el PTLC para seguir ejerciendo sus funciones de transferencia y promoción de empresas de base tecnológica en los años venideros.

\section{Definición de Parque TeCnológico}

Actualmente no existe en la literatura especializada una definición globalmente aceptada respecto a la noción de Parque Científico Tecnológico (PCT). Esto se debe a que los parques han sido un espacio productivo, científico y técnico que ha mutado a lo largo de los años y que ha experimentado cambios de interpretación fruto de los tiempos, las condiciones culturales, políticas, tecnológicas y socioeconómicas de los países.

En este sentido, pueden encontrarse definiciones que resaltan en mayor o menor medida algún aspecto que consideran fundamental de este tipo de aglomeraciones: aquellas que exaltan la cuestión de la asociatividad, las que hacen hincapié en la transferencia de conocimiento y las más escépticas, que priorizan la cuestión relativa a la valorización del espacio urbano. Finalmente, se destacan las que buscan abarcar al parque en su complejidad, destacando todos los aspectos que hacen de estos espacios un ámbito dinamizador de la actividad económica local o regional.

De acuerdo a Rodríguez Pose (2012), la mayoría de los académicos reconoce a los PCT como una iniciativa -generalmente pública- de creación de un área geográfica delimitada y destinada a favorecer el desarrollo y la aplicación de actividades científicas y tecnológicas. Esta área tiene como fin promover y albergar instituciones de investigación y empresas intensivas en el uso y la generación de conocimiento, entre las que se estimula la producción y la transferencia. Esta transferencia se da principalmente dentro del área del parque y en su entorno, pero también con instituciones de investigación y empresas localizadas fuera de él.

Además, el estímulo para esta actividad ocurre mediante una gestión activa del área por parte de profesionales especializados, ofreciendo a centros de investigación y empresas servicios de alto valor añadido, así como espacio físico y servicios básicos.

El objetivo último de toda esta actividad es la generación de crecimiento económico sostenible en el largo plazo dentro del territorio (Rodríguez-Pose, 2012). En esta misma línea argumental, la APTE (1999) define a un Parque Científico y Tecnológico como un lugar dotado de servicios avanzados donde se ubican empresas, que tienen lazos formales $\mathrm{u}$ operacionales con una universidad, un centro de investigación o una institución de educación superior.

Desde el punto de vista de esta organización, el parque está diseñado para fomentar la creación de industrias basadas en el conocimiento y de empresas de alto valor añadido del sector terciario. Además, él mismo cuenta con un equipo de gestión que ánima los fenómenos de transferencia de tecnología y fomenta la mejora de la competitividad de las empresas que allí se ubican.

Por su parte, para la International Asociation of Science Parks, un Parque Científico constituye una entidad gestionada por profesionales especializados, cuyo objetivo fundamental es incrementar la riqueza de su comunidad, promoviendo la cultura de la innovación y la competitividad de las compañías e instituciones generadoras de saber instaladas en el parque o asociadas a él (Adán, 2012). Con este 
propósito, la estructura estimula y gestiona el flujo de conocimiento y tecnología entre universidades, instituciones de investigación, empresas y mercados; impulsa la creación y el crecimiento de firmas innovadoras mediante mecanismos de incubación y de generación centrífuga (spin-off) y proporciona otros servicios de valor añadido, así como espacio e instalaciones de gran calidad.

Finalmente, Doreen Massey y otros (1992) resumen adecuadamente los diferentes objetivos que presentan los parques científicos y tecnológicos (tabla 1). Desde su óptica estos objetivos son: a) desarrollo económico; b) transferencia de tecnología; c) generación de beneficios a escala local.

Tabla 1. Objetivos de los parques científicos y tecnológicos.

\section{Desarrollo Económico}

Estímulo a la formación de empresas de base tecnológica

Promoción de las empresas de base tecnológica existentes

Comercialización de la investigación académica aplicada

Desarrollo de las tecnologías del futuro

Contrarrestar el desbalance regional en capacidades de I+D, inversión e innovación Atraer inversión externa y movilizar I+D

Transferencia de Tecnología

Promover spin-off desarrollados por personal académico

Promover vínculos entre la universidad y la industria

Facilitar la transferencia de tecnología de la universidad a la industria

Darle a las instituciones académicas acceso a modos de comercialización de I+D

Incrementar la apreciación que los académicos tienen respecto de las necesidades industriales

Estimular innovaciones basadas en conocimiento científico

Beneficios a escala local

Crear trabajo y oportunidades de consultoría para académicos y estudiantes

Crear sinergia entre empresas

Generar nuevos empleos

Incrementar la performance de la economía local

Estimular cambios en la imagen del territorio

Desarrollar confianza en las capacidades locales

Impulsar una cultura emprendedora

Generar ingresos para las instituciones académicas

Mejorar la imagen de las instituciones académicas en los ojos del gobierno central

Fuente: traducción propia en base a Massey y otros (1992).

El énfasis puesto en cada tipo de objetivo depende las demandas sociales y el target de la audiencia a la que se dirigen las autoridades del parque: el énfasis en los beneficios locales, por ejemplo, puede hacerse cuando los promotores del emprendimiento se dirigen a la población que será objeto de la implantación de la iniciativa. Por su parte, el foco en la transferencia de tecnología se pone en la discusión que se realiza con instituciones educativas y científicas, mientras que el eje 
en el desarrollo económico se da cuando el proyecto se presenta ante el gobierno central.

A su vez, estos objetivos también difieren de acuerdo a las condiciones locales en las que se plantea la creación del PCT: en áreas que están atravesando una situación de declive, particularmente en los casos de áreas industriales antiguas, la conformación de un parque tecnológico puede significar numerosos beneficios locales en lo que respecta a la creación de empleo y en el desarrollo de nuevas empresas de base tecnológica. En otros casos en los que la universidad tiene un peso muy significativo en la comunidad, los objetivos principales pueden pasar por la transferencia de tecnología y por desarrollar sinergias entre la academia y la industria.

Según Massey y otros (1992) es debido a esta multiplicidad de objetivos (que pueden destacarse en mayor o menor medida) que no existe una definición unívoca del término parque tecnológico: esto nos da la pauta de que cada organización de esta naturaleza es en cierta medida singular y presenta su propia impronta.

\section{ANTECEDENTES}

De acuerdo a Adán (2012), existen más de 400 parques científico-tecnológicos en el mundo. El origen de los mismos se encuentra en América del Norte, creándose luego en Europa, posteriormente en Asia y finalmente en América Latina. A continuación, se presenta una breve síntesis de las experiencias más importantes registradas en cada uno de los continentes.

\subsection{América del Norte}

La primera experiencia de Parque Tecnológico exitoso a nivel mundial está asociada a Estados Unidos, específicamente al Silicon Valley o Valle del Silicio ${ }^{2}$.

Este parque, desarrollado poco después del término de la segunda guerra mundial, está situado al sur de la bahía de San Francisco en California. Sus principales promotores fueron William Shockley y Frederick Terman (ambos miembros del cuerpo docente de la Universidad de Stanford). Allí se logró relación, cooperación y alianza para el desarrollo de tecnología entre distintos sectores de la sociedad: universidad, industria y gobierno (Saxenian, 1990).

El modelo denominado Triple Hélice de Etzkowitz y Leyersdorf (1966) establece que estas relaciones llevan a un proceso eficiente de coordinación que permite generar innovación. Precisamente a partir de estos vínculos generados y de otros factores idiosincráticos y culturales, este tipo de emprendimiento alcanzó éxito.

Si bien, los distintos mundos (el científico, académico y empresarial) no poseen los mismos intereses, en este espacio se generó una sinergia entre ellos cuyo objetivo fue transferir tecnología.

Ocurrió que la universidad estaba interesada en el desarrollo económico, existía cooperación con empresas e implementación de otras en los establecimientos universitarios, la existencia de una demanda estimuló la innovación, la presencia de un mercado financiero hizo posible las negociaciones y además el rol del gobierno

\footnotetext{
${ }^{2}$ El nombre del parque se debe a que en sus inicios varias empresas de chips de silicio se asentaron ahí.
} 
con implementación de políticas, favoreció el desarrollo del parque y de toda su área de influencia (Saxenian, 1991)3.

En el Silicon Valley puede encontrarse aglomeraciones de empresas dedicadas a la fabricación de semiconductores, procesadores, computadoras, nanotecnología, software, biomedicina y tecnologías de la información y la comunicación. Actualmente en el Silicon Valley se genera el 9,2\% del empleo total de California (1.423.491 puestos de trabajo), siendo el sueldo anual promedio de un empleado de $\$ 107.395$ dólares anuales, muy por encima del promedio estatal. A su vez, la economía del Valle del silicio aporta al Producto Bruto Geográfico de California el 9,9\% del valor añadido global (Silicon Valley Institute for Regional Studies, 2014).

Un dato que resulta de interés remarcar es que los niveles de innovación de este territorio son sorprendentes, medidos por cualquier estándar y lideran la escala nacional de los Estados Unidos. Para el año 2012, se habían registrado un total de 15.057 nuevas patentes y la inversión en ángeles había superado los 507 millones de dólares (Silicon Valley Institute for Regional Studies, 2013).También la economía del valle del Silicio lidera la creación de empleos a nivel país, generando aproximadamente 46.000 trabajos anuales, siendo el nivel de desempleo del territorio del orden del $5 \%$, lo que puede considerarse una tasa de desempleo friccional (Silicon Valley Institutefor Regional Studies, 2014).

En el caso de Estados Unidos, han surgido implantaciones exitosas de parques temáticos en Seattle, donde se concentran actividades bioquímicas y de software y en Mineápolis, con desarrollos significativos en el área de los semiconductores, entre otros lugares.

Por otro lado, en Canadá los primeros polos Científico-Tecnológicos se crean durante la década de 1980. Específicamente, estos parques se encuentran distribuidos en forma heterogénea entre 8 provincias y 13 aglomeraciones urbanas, exhibiendo distintas configuraciones: existiendo los de naturaleza pública, los de tipo privado y los de carácter mixto (Shearmour y Doloreaux, 2000).Las áreas de interés de los mismos son diversas, destacándose los rubros aeronáutica y aeroespacial, farmacéutica, biotecnología, agricultura y procesamiento de alimentos, tecnologías de la información, microelectrónica y equipamiento informático.

Entre los más destacados se encuentran el Metropolitan High Tech Park de Quebec, el Discovery Science Park de Vancouver y el Sheridan Science and Technology Park de Missisauga (Shearmour y Doloreaux, 2000).

\subsection{Europa}

Durante el transcurso de los años ochenta, en el viejo continente las universidades fueron quienes impulsaron la creación de parques, los cuales se estructuraron como sociedades anónimas. El objetivo de las casas de estudio era obtener una rentabilidad económica a partir de los conocimientos científicos. Específicamente, esta experiencia fue típica de países como Escocia e Inglaterra.

En Gran Bretaña, se crearon polos tecnológicos adyacentes a los campus universitarios, tal es el caso Cambridge, Herriot-Watt en Edimburgo y el Manchester

3 Las sinergias que se generaron provocaron crecimiento de aglomeraciones industriales y de otros parques de ciencia y tecnología en localidades vecinas como Mountain View, SunnyVale, San José y Cupertino. 
Science Park, respectivamente. Existen indicadores que dan cuenta del éxito obtenido por los mismos, como el aumento de inversiones, el incremento de empresas de base tecnológica, la mayor tasa de empleo y la generación de una fuerza de trabajo especializada.

En el año 1975 se funda el Parque Científico de Cambridge, el cual adquiere notoriedad diez años más tarde, a partir de un estudio denominado "el fenómeno de Cambridge". Este trabajo, además de establecer las características de la iniciativa, fue importante porque le dio relevancia a las actividades de alta tecnología (Benko, 1998).

Por su parte, Herriot-Watt en Edimburgo, también se caracterizó por contar con un importante patrimonio inmobiliario, que sirvió como un elemento extra de valorización para las empresas, además de la transferencia y la comercialización de tecnología.

Por otro lado, Manchester Science Park fue creado a finales de los años 8o. En sus más de 25 años de historia ha apoyado el crecimiento de empresas innovadoras, principalmente en los sectores de $\mathrm{TIC}^{\prime} \mathrm{s}$, biotecnología, tecnologías industriales y digital media. En la actualidad este tecnopolo está integrado en el Corredor Tecnológico de la ciudad de Manchester, que destina más de 2.500.00o metros cuadrados de la ciudad a potenciar sectores intensivos en conocimiento. En esta área se ubican las dos universidades de la ciudad (la Universidad de Manchester y la Universidad Metropolitana) y la Central de Hospitales (Central Manchester University Hospitals) (Adán, 2012).

Como un caso atípico al resto de los existentes en Europa, cabe mencionar el de Sophia Antípolis en Francia. Este parque se crea sin contar con una Universidad cercana y en un contexto de escasa actividad empresarial. En la literatura especializada se establece que su éxito se genera a partir de su privilegiada situación geográfica y motivo de una exitosa estrategia pública que incentivó la radicación de empresas, en el marco de una política nacional de descentralización de la actividad económica y científica.

En términos generales, la misma consistía en incentivos para trasladar laboratorios, equipos, y personal de las universidades y otras instituciones de enseñanzas a regiones de la periferia. De esta manera, estas zonas se veían favorecidas con una oportunidad para el desarrollo tecnológico.

Como bien plantea Ondategui (2001), el fenómeno francés persigue el crecimiento de ciudades medianas en áreas no metropolitanas a partir del impulso de funciones de comando de carácter industrial y de servicios avanzados. De acuerdo a Chicoye (1989), en un lapso de 20 años el tecnopolo Sophia Antipolís generó 10.000 puestos de trabajo en forma directa y un total de 25.00o indirectos, provocando que un 10\% de la población económicamente activa de la provincia viva del o alrededor de este emprendimiento.

Una situación similar ocurre en otras localidades medias de Francia, como por ejemplo Lyon, Grenoble, Toulouse y Montepellier. En esta última ciudad se encuentran cinco tecnopolos especializados: Euromedicine ligado a la sanidad, Agropolís, especializado en los recursos de la tierra, Anntena en multimedia, Communicatique en informática y robótica y finalmente, Helipolis en turismo y actividades recreativas (Malezieux, 1993). 


\subsection{Asia}

En Asia, Singapur fue uno de los primeros países en impulsar empresas de base tecnológica a partir de la creación del Singapore Science Park. En relación a esta cuestión, Ondategui (2001) manifiesta que:

Durante los años 90 Singapur se destaca por su industria de alta tecnología (reparación de aviones, equipos electrónicos y servicios avanzados) que constituyen el $75 \%$ del PBI. Su puerto con excelentes instalaciones para el tráfico de contenedores, reexporta productos refinados del petróleo para los países del área y bienes de equipo para todo el mundo (Ondategui, 2001, p. 104).

El Singapour Science Park nace como un esfuerzo coordinado del gobierno en materia de ciencia y tecnología. La inversión pública incluyó la formación de recursos humanos, la construcción de infraestructura tecnológica y más recientemente la promoción de emprendimientos (Koh et al, 2005).

En este país la estrategia de conformación de tecnopolos se apoya en la confluencia entre política pública y redes de empresas de alta tecnología de carácter mundial, como Conner Peripherals, Hewelet Packard y Thomson-SGS.

Para complementar al Singapour Science Park, el gobierno está construyendo un nuevo polo denominado One North Science Habitat, un área de 200 hectáreas perfectamente equipada en la cual confluirán universidades y empresas para el desarrollo de la biotecnología (Koh et al, 2005).

Por su parte, Tsukuba Science City fue el primer parque construido en los años 80 en Japón. En 1960 se estableció la idea de crearlo, diez años más tarde comenzó su construcción y recién en 1980 empezó a funcionar. A partir de la década del 90, el estado Japonés desarrolla una política agresiva de impulso de la ciencia y la tecnología que permitió que el país alcance un número de 158 parques para el año 1998 (Park, 2001).

Debido a esta cuestión, la literatura especializada sostiene que el desarrollo tecnológico ha sido una de las variables más significativas para explicar el crecimiento económico del país y la profunda transformación de su estructura productiva.

Finalmente, en China también se ha registrado un importante crecimiento de parques tecnológicos de diversa índole. Específicamente en Taiwán se ha erigido el parque Hsinchu, una combinación entre una zona franca de exportación y un polo tecnológico, que contiene en su seno más de 150 empresas -principalmente de las ramas de las telecomunicaciones y de la industria computacional-; entre ellas se encuentra la firma Hacer Computers, una multinacional que a inicios de la década del 80 era apenas una pequeña empresa (Barbieri, 1994). Otros de los parques más significativos del gigante asiático son: Z-Park, Hong Kong Science Park y SIPIS-Suzhou.

\subsection{América Latina}

En Latinoamérica los primeros parques científico-tecnológicos se implementan recién en la primera década del siglo XXI. Este proceso ocurrió más tarde que en los países desarrollados, debido a las particularidades propias del 
contexto latinoamericano, el cual difiere de los países anteriormente mencionados. Según Carlos Botella e Ignacio Suárez (2012), la tardía difusión de la segunda revolución industrial en la región tuvo como consecuencia un atraso tecnológico respecto a las regiones más desarrolladas.

Se debe aclarar que los países subdesarrollados presentan problemas estructurales. Algunos de ellos son: recursos escasos, inflación, desempleo, debilidad en los marcos institucionales, inestabilidad política y económica, falta de inversiones extranjeras e infraestructura obsoleta. Estas condiciones provocan que en el sector de Innovación y Tecnología no se elaboren políticas consistentes que favorezcan la creación y desarrollo de parques de esta naturaleza. Respecto a esta cuestión, Rodríguez Pose (2012) sostiene que:

Se puede decir que, aunque la participación del sector privado ha sido una constante en los PCT latinoamericanos, su papel ha sido de menor importancia en comparación con un porcentaje significativo de los parques europeos, y sobre todo, de los Estados Unidos, siendo su nivel de financiación privada muy escasa. La financiación de los PCT en América Latina ha sido mayoritariamente de carácter público, pero la falta de recursos ha hecho que una parte importante de los PCT identificados estén, o hayan estado durante años en fase de proyecto. (Rodríguez Pose, 2012, p. 30).

Los inicios de este proceso datan de 1984, con la creación del Programa Brasileño de Parques Científicos. Se reconoce a Brasil como el país de América Latina con más desarrollo en esta temática. A mediados de los años 1980, se intentan las primeras iniciativas, cuando no estaban dadas aún las condiciones necesarias para su desarrollo y en medio de una crisis macroeconómica de importantes dimensiones.

Hasta el año 2008, en Brasil había 74 parques científico tecnológicos, que acompañaban alrededor de 6300 empresas y generaban 33.000 empleos directos (Lahorgue, 2010). La gran mayoría de los parques operativos se localizan en los estados del sudeste y del sur, los de mayor ingreso per cápita del país, los de mayor tradición industrial y recursos humanos calificados. Gran parte de los parques en estos estados se encuentran en grandes aglomeraciones urbanas o en sus alrededores. En particular se destacan los tres parques científicos tecnológicos de Río de Janeiro, los dos existentes en Porto Alegre y el parque Ciatec Campinas, uno de los más grandes y dinámicos del territorio brasileño4.

Brasil es seguido por México como el país con mayor implantación de Parques Científicos y Tecnológicos en América Latina. En México se reconocen un total de 35 parques, de los cuales 21 están operativos, siete en proceso de implantación y otros siete en proyecto. De todos los parques identificados, el más ambicioso es el Parque Tecnológico Silicon Border de Mexicali, Baja California. El mismo consiste en un ámbito especializado en semiconductores y energías, con énfasis en el desarrollo de energía solar. Cuenta con 1800 hectáreas y puede crecer hasta 4000, pudiendo aprovechar las ventajas de la Frontera con los Estados Unidos y la cercanía con polos dinámicos como California o San Diego y la aglomeración de Los Ángeles (Rodríguez-Pose, 2012).

\footnotetext{
4 En este último parque se puede encontrar empresas de los sectores de tecnologías de la información y la comunicación, energía, medio ambiente, cosmética, ingeniería industrial y biotecnología.
} 
En Argentina las primeras iniciativas de esta naturaleza datan de la década de los 90, principalmente en Córdoba. Originalmente no adoptaron la forma de Parques Científicos, sino la de incubadoras de empresas que luego han ido ampliando su oferta de servicios. En comparación a los parques brasileros, existe en este país un número mucho menor de emprendimientos de esta naturaleza y a su vez el tamaño y los servicios que los mismos prestan difieren.

En este sentido, existen variaciones en el tamaño de las iniciativas, con respecto al número de empresas que engloba cada parque, en lo relativo al espacio físico y a la cantidad de empleados que genera cada emprendimiento. De acuerdo a Rodríguez Pose (2012), pueden reconocer en Argentina un total de cinco parques operativos, uno en proceso de implantación y uno en proyecto. El que ha adquirido más desarrollo es el Parque Tecnológico Litoral Centro (PTLC).

\section{Parque Tecnológico litoral Centro}

\subsection{Ubicación, historia de su creación y forma de organización}

El Parque Tecnológico del Litoral Centro, se encuentra radicado en la Provincia de Santa Fe (Argentina), sobre la ruta Nacional 168. El mismo está ubicado en una zona privilegiada, integrando el corredor Coquimbo-Puerto Alegre, en el centro de las vías de comunicación terrestres que unen el MERCOSUR.

Este posicionamiento estratégico hace que el parque participe de un gran circuito comercial, tanto local como regional. A su vez, este polo tecnológico se encuentra a 2 kilómetros del centro de la ciudad de Santa Fe, a 5 kilómetros del aeropuerto, y a $2 \mathrm{~km}$ de la autopista que lleva a la ciudad de Buenos Aires. En este sentido, su ubicación posibilita que tanto empresarios como investigadores asociados a la institución tengan un rápido acceso a distintos centros, tanto financieros, educativos, como de consumo y recreación (Cellino, 2012).

La idea de crear el Parque Tecnológico del Litoral Centro (PTLC) se delinea en la década del noventa, con el objetivo de brindar una solución a las demandas del empresariado santafesino que buscaba aliarse al sector científico para responder a las exigencias productivas de un mercado cada vez más complejo. En un contexto de creciente incorporación de tecnología a los procesos productivos y de valor a la producción, la innovación se transforma en el factor productivo por excelencia, para lograr ser competitivo en el contexto de la globalización.

La viabilidad del proyecto tiene su fundamento en la experiencia favorable que poseían estos emprendimientos en Estados Unidos y Europa y en la existencia, a nivel local, de una alta proporción de investigadores por habitante.

Convencidos de las condiciones inmejorables que presentaba Santa Fe y la región para el logro de los objetivos planteados, el Consejo Nacional de Investigaciones Científicas y Técnicas (CONICET) decidió crear el Parque Tecnológico del Litoral Centro (PTLC). El proyecto no tardó en concretarse debido a que el organismo nacional ya poseía los terrenos para su emplazamiento. Los mismos habían sido donados por la Municipalidad al INTEC (Instituto de Desarrollo Tecnológico para la Industria Química) 5 en el año 1975. A su vez, ya se contaba con

\footnotetext{
5 Instituto de doble dependencia CONICET y Universidad Nacional del Litoral.
} 
infraestructura edilicia, que por aquel entonces, había comenzado a erigirse para albergar y aglutinar la oferta científica local.

En 2001 el CONICET impulsó y aprobó la constitución de una Sociedad Anónima encargada de administrar el área de incubación y los predios para radicación de Empresas del PTLC. Tomando como modelo la figura jurídica de los Parques Tecnológicos de Andalucía y Galicia, se decidió crear una Sociedad Anónima con Participación Estatal Mayoritaria (S.A.P.E.M.), en donde interviniesen de manera equilibrada los sectores científico, gubernamental y empresarial.

El Consejo Nacional de Investigaciones Científicas y Técnicas (CONICET), la Universidad Nacional del Litoral (UNL), el Gobierno de la Provincia de Santa Fe, la Municipalidad de la ciudad de Santa Fe, la Confederación General Económica (CGE) y la Confederación General de la Industria (CGI) son los socios fundadores del Parque Tecnológico del Litoral Centro S.A.P.E.M. Esta sociedad, que adquirió personería jurídica el 22 de agosto de 2002, incorporó dos años después un nuevo accionista: la Municipalidad de la ciudad de Paraná (Entre Ríos).

La forma jurídica de sociedad anónima con participación estatal mayoritaria, implica que el estado se reserva la mayoría accionaria, contando con un $51 \%$ de dicho capital.

Dentro del paquete accionario de la sociedad se reconocen tres tipos de acciones: la clase A, la clase B y las C. El total de acciones emitidas por la sociedad es de 12.000, encontrándose distribuidas de la siguiente forma: clase A: 4.080 acciones; clase B: 3096; clase C: 3096.

Las acciones clase A se encuentran en poder del sector científico-tecnológico. Originalmente se encontraban solo en manos de la Universidad Nacional del Litoral, ya que el CONICET tenía prohibido por estatuto integrar Sociedad Anónimas, situación que se regularizó hace dos años a partir de un decreto emitido por el Poder Ejecutivo Nacional. Actualmente, el 34\% del paquete accionario está en manos de estas dos entidades.

De acuerdo al estatuto de conformación de la sociedad, la presidencia de la entidad queda reservada exclusivamente a un miembro de este grupo, el cual debe cumplir necesariamente con un doble requisito: ser profesor de la Universidad del Litoral y, simultáneamente, investigador del CONICET.

Las acciones clase B corresponden al sector gubernamental, integrado actualmente por el gobierno Provincial de Santa Fe, el del Municipio Homónimo y el de la Municipalidad de Paraná.

Por su parte, las acciones clase $\mathrm{C}$ son propiedad de las entidades gremiales empresarias: la Confederación General de la Industria y la Confederación General Económica. A su vez, el estatuto prevé que las firmas radicadas en el parque puedan acceder a estos papeles, una vez que inicien sus actividades productivas dentro del parque.

Tal y como sucede con este tipo de sociedades en cualquier parte del mundo, la misma consta de un directorio, integrado por nueve miembros. Este órgano de gobierno está conformado por tres representantes de los accionistas clase A (con sus respectivos suplentes), tres representantes del grupo clase $\mathrm{B}$ y tres dirigentes correspondientes al segmento clase C (Gráfico 1). 
Gráfico 1. Organigrama del Parque Tecnológico Litoral Centro.

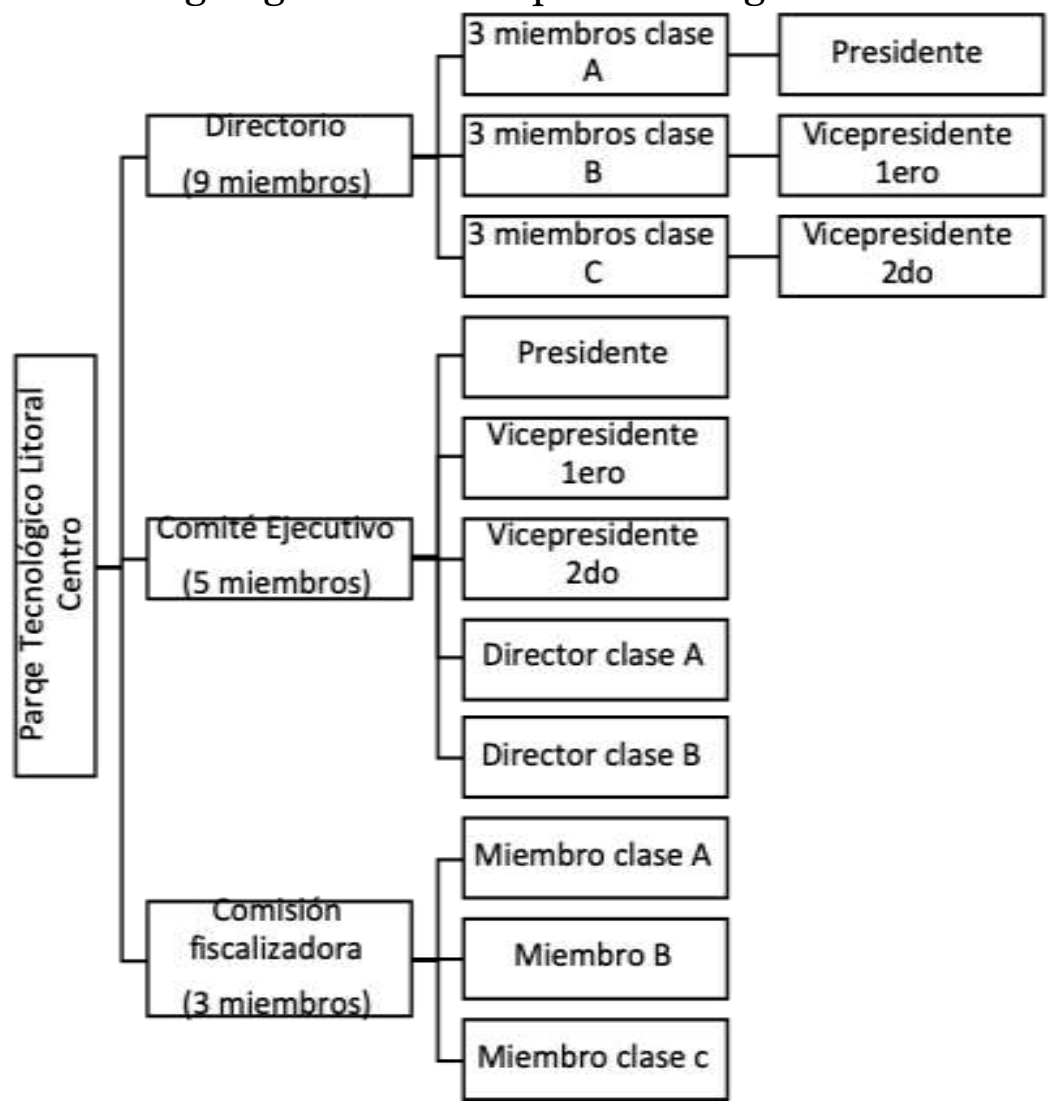

Fuente: Elaboración propia.

El Directorio constituye el máximo órgano de representación de la entidad y solo se convoca ante situaciones extraordinarias, como por ejemplo, la necesidad de una modificación del estatuto. Para garantizar el normal funcionamiento del parque y el desenvolvimiento de todas sus actividades, la sociedad dispone de un comité ejecutivo, que es el encargado de garantizar la operatividad diaria.

Este comité ejecutivo está integrado por cinco miembros: un presidente primero; un vicepresidente; un vicepresidente segundo; un miembro nombrado por los accionistas clase A y un representante nombrado por los accionistas clase B. Finalmente, la entidad cuenta también con un órgano fiscalizador, encargado de monitorear las finanzas de la organización y la transparencia en el ejercicio de sus funciones. Esta comisión también está conformada por representantes de todas los segmentos accionarios, cada miembro con su respectivo suplente.

\subsection{Principales actividades}

A partir del año 2003, luego de haber logrado personería jurídica, el PTLC intensifica notablemente sus actividades, conformando diferentes áreas de trabajo y esferas de actuación. Hoy en día el PTLC lleva construidos $7500 \mathrm{~m}^{2}$ de edificios, destinados a laboratorios, oficinas, espacios comunes y otras obras de infraestructura.

A su vez, se han generado dentro del parque un total de 315 puestos de trabajo, existen 47 emprendimientos que fueron atendidos por la organización y se han capacitado a un total de 1050 personas (Cellino, 2012). 
Además, el PTLC se ha transformado en un ente capaz de incentivar notablemente las exportaciones con alto valor agregado de la localidad de Santa $\mathrm{Fe}$ y su zona de influencia. De acuerdo a datos suministrados por la Provincia, el monto exportado por el Parque en el año 2010 ascendía a 42.499 millones de dólares, siendo el valor medio de exportación por tonelada de 1661,7 dólares, muy superior al promedio provincial (Cellino, 2013).

A continuación, se detallan las principales acciones desarrollas por el Parque Científico Tecnológico Litoral Centro.

\subsection{Dictado de cursos y actividades de difusión}

Desde el año 2002, el PTLC realiza actividades formativas y de difusión tanto de sus acciones como de los alcances y objetivos que tienen los parques tecnológicos en el mundo. La mayoría de este tipo de iniciativas ha estado a cargo de su director, el Dr. Amadeo Cellino, que ha realizado conferencias y disertaciones sobre la temática en el país y en el extranjero.

A su vez, el PTLC ha generado un curso sobre desarrollo emprendedor, que se dicta anualmente en la Facultad de Bioquímica de la Universidad Nacional del Litoral. Originariamente, el curso fue pensado para el personal docente de la Facultad, pero luego se abrió a toda la comunidad educativa y al público en general. Este curso,a partir del año 2004, se generó en forma conjunta con la Secretaría de Vinculación Tecnológica y Desarrollo Productivo de la Universidad Nacional del Litoral (UNL), desarrollándose posteriormente el Programa de Emprendedores.

Esta propuesta tiene como objetivo promover y apoyar toda idea o iniciativa emprendedora nacida de estudiantes o graduados que estimule el espíritu empresarial en la región y aliente la creación de firmas de base tecnológica y productivas innovadoras, sobre todo entre los jóvenes (Universidad Nacional del Litoral, 2012b). En el marco de este programa se estableció una cátedra electiva que puede ser tomada por estudiantes de cualquier facultad y que se conformó en base a los contenidos de dicho curso, originalmente creado por integrantes del PTLC.

A su vez, el PTLC también ha colaborado con la Secretaría de Vinculación y Desarrollo Productivo de la UNL en la conformación de gabinetes de desarrollo emprendedor, que funcionan en las distintas facultades, y que tienen como principal objetivo brindar un primer asesoramiento a todos los estudiantes que quieran iniciarse en la actividad empresarial (Universidad Nacional del Litoral, 2012b).

Finalmente, este proyecto también cuenta con un página web, un blog, una página de Facebook, un programa de radio y organiza una serie de Jornadas Anuales para difundir ideas de proyectos desarrolladas por los propios estudiantes. Hasta el año 2012, un total de 96 iniciativas contaron con el asesoramiento de dicho programa (Universidad Nacional del Litoral, 2012a).

\subsection{Incubación de Empresas}

Además de la formación de emprendedores, el PTLC presenta instalaciones que permiten que una empresa desarrolle todo el ciclo de negocio dentro de su espacio físico. Específicamente dentro de esta área de trabajo se pueden reconocer diferentes secciones, a saber: a) preincubación; b) incubación; c) prerradicación y d) radicación. 
Cualquier firma que entra en contacto con el PTLC puede incorporarse en cualquiera de las etapas enunciadas anteriormente, en función del grado de avance que tenga su idea de negocio o proyecto. A lo largo de todo el ciclo de vida del negocio, el PTLC se compromete a mantener una estricta confidencialidad sobre la actividad desarrollada por la firma y a trabajar en la protección de su proceso de "saber hacer" (Gráfico 2).

Gráfico 2. Secuencia del Ciclo de Negocio en el PTLC.

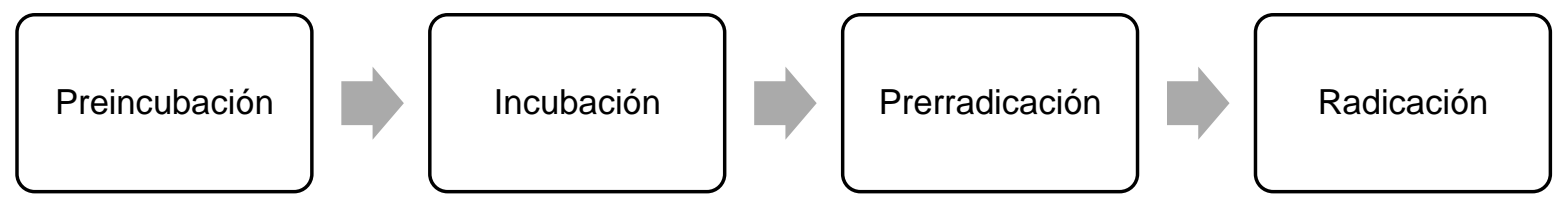

Fuente: elaboración propia.

La etapa de preincubación está reservada para aquellas personas físicas o sociedades que se encuentran en la fase inicial o de conformación del emprendimiento. Aquí se colabora con los emprendedores en el diseño y elaboración del plan de negocios, es decir, la transformación de una idea en una alternativa productiva o de servicios. El sistema de preincubación del PTLC S.A.P.E.M. proporciona a los emprendedores puestos de trabajo provistos con computadoras conectadas a internet, equipamiento para el desarrollo de sus actividades (sala de reuniones multimedia, fotocopiadora. fax e impresora), además de asesoramiento en las áreas tecnológica, de propiedad intelectual, jurídica, contable y financiera.

Actualmente el PTLC cuenta con dos ideas de negocios preincubadas, siendo la más importante una dedicada a la producción de biodisel a partir de microalgas oleginosas. A su vez, existen 5 pedidos depre incubación que se encuentran en etapa de evaluación y que corresponden a las siguientes ideas-negocio: $1^{\circ}$ ) Desarrollo de una vacuna de $\mathrm{ADN}$ contra el virus de la Anemia infecciosa Equina; $2^{\circ}$ ) Producción de bioetanol a partir de desechos industriales; $3^{\circ}$ ) Fabricación de una máquina hortícola multipropósito y Agroecológica; $4^{\circ}$ ) Prestación de un servicio de control y gestión de bebidas alcohólicas; $5^{\circ}$ ) Elaboración de un proceso de Embotellamiento para bebidas no gasificadas, que utiliza un sistema de máquinas expendedoras electrónicas.

Por su parte, la fase de incubación constituye un ambiente acondicionado para favorecer el surgimiento y maduración de las iniciativas empresariales. La incubadora de empresas del PTLC brinda a cada firma en régimen de alquiler un espacio físico modular acorde a sus necesidades: laboratorios de $45 \mathrm{~m}^{2}$ con pileta de desagüe para líquidos especiales, suministro de gas, tres terminales de computación y doce tomas de red independientes para computación y gabinetes de $46 \mathrm{~m}^{2}$. Estos nuevos servicios se suman a los esenciales, ya suministrados en la etapa previa. De acuerdo a la normativa establecida por el parque, una empresa puede permanecer en la fase de incubación por un período de dos años, aunque existe la posibilidad de prórroga. 
En este etapa de la cadena hay 12 firmas establecidas entre las que se destacan las siguientes: $1^{\circ}$ ) BIOPARX: especializada en fabricación de prótesis y ortesis; $2^{\circ}$ ) ASSO NETWORK SOLUTIONS: firma dedicada a la instalación, mantenimiento y soporte de redes de servicios informáticos; $3^{\circ}$ ) DINT S.A: empresa especializada en sistemas de monitoreo remoto para Detención Domiciliaria, Rastreo y Seguimiento; $4^{\circ}$ ) FICHEM: fabricante de compuestos químicos para uso odontológico; $5^{\circ}$ ) PROTECH FARMA SA: dedicada a la producción de sustancias químicas medicinales y de uso botánico; $6^{\circ}$ ) LIPOMIZE SRL: dedicada a la fabricación de nano soluciones liposómicas para la industria farmacéutica, cosmecética y nutricional $6 ; 7^{\circ}$ ) NOVARTEK: dedicada a la producción y comercialización de microcápsulas inyectables biocompatibles y bioasimilables que permiten la liberación sincronizada de hormonas inductoras de celo y ovulación.

En lo que respecta al área de prerradicación del PTLC, la misma se encuentra diseñada a imagen y semejanza del modelo europeo de contenedores de empresas y se creó para brindar a las firmas egresadas de la incubadora la infraestructura elemental para que pudiesen iniciar la fase productiva. Dentro de dichos módulos -de 100 metros cuadrados cada uno- los emprendedores pueden construir las dependencias (oficinas, laboratorios y/o vestuarios) inherentes a la actividad económica de la firma y a las necesidades funcionales del proyecto. También pueden edificar dependencias auxiliares fuera del contenedor, pero respetando las características constructivas fijadas por la S.A.P.E.M.

Al finalizar el período de prerradicación, si la empresa demuestra que ha podido afianzarse técnica y económicamente y declara su necesidad de seguir vinculada al sector científico y tecnológico, podrá solicitar predios para radicarse definitivamente en el PTLC. Hoy día se encuentran formando parte de la fase de prerradicación dos firmas: HORIAN CARBONFE e INCUBATECH SA.

La primera de ellas se dedica a la fabricación y preparación de biofármacos, los cuales se mejoran a través de procesos de pegilación. La segunda empresa en cambio, se encuentra especializada en la producción de compuestos activos para medicamentos biogenéricos desarrollados a través de biotecnología, entre los que se encuentran la hormona de crecimiento humana recombinante (rgh-somotropina) utilizada en diversas prácticas médicas.

Por su parte, el Área de Radicación Industrial es la última fase del modelo de desarrollo empresarial. La misma consta de un predio de 14 hectáreas de campo propiedad del CONICET, que son administradas por el Parque Tecnológico Litoral Centro S.A.P.E.M. y otorgadas a las firmas mediante un contrato de "cesión de uso" por 30 años.

$\mathrm{Al}$ establecerse en este predio, las compañías obtienen beneficios impositivos, además de una serie de servicios que garantizan una adecuada preservación de las instalaciones construidas por la empresa: alambrado perimetral y seguridad privada,

${ }^{6}$ En el año 2012, la firma LIPOMIZE SRL obtuvo los siguientes reconocimientos: Subsidio Pre-semilla otorgado por la Fundación Argentina de Nanotecnología (FAN); Beca de Innovación Tecnológica otorgada por la Fundación Nuevo Banco de Santa Fe; Capital semilla otorgado por el Ministerio de Industria de la Nación; 1er Premio en el concurso "Una Apuesta al Tricentenario" organizado por la Bolsa de Comercio de Santa Fe; 1er Premio en el concurso "Emprendimientos Innovadores Banco Nación”organizado por la Fundación EMPRETEC; 1er Premio en el concurso "Jóvenes Emprendedores Santander Río". 
entre otros. En este espacio, El PTLC se reserva el derecho de fijar normas de edificación, a fin de conservar el equilibrio entre naturaleza y arquitectura, mientras que las firmas se ven obligadas a invertir el $4 \%$ de sus utilidades en actividades de investigación y desarrollo. Estas actividades de I+D deberán ser contratadas al PTLC, al CONICET o en su defecto a la Universidad Nacional del Litoral, siempre y cuando el servicio que puedan prestar las entidades sea acorde a las necesidades de la empresa y se preste a un precio razonable en relación al fijado por la competencia.

En esta sección del parque se encuentran actualmente establecidas tres empresas, de las cuales se destacan fundamentalmente dos: ZELTEC y ZOOVET (PRODUCTOS VETERINARIOS SA). La firma ZELTEC es una empresa del grupo AMEGA BIOTERCH y constituye un spin off tecnológico de la Universidad Nacional del Litoral, realizado por Ricardo Kratje y Marina Etcheverrigaray, directores del Laboratorio de Cultivos Celulares de la Facultad de Bioquímica y Ciencias Biológicas.

ZELTEC posee dentro del parque una planta industrial de tres pisos, con $1.700 \mathrm{~m}^{2}$ cada uno, dedicada a la producción de diversos fármacos, entre los que se destaca la eritropoyetina humana recombinante, una hormona necesaria para la producción de glóbulos rojos, que se suministra a los enfermos renales.

Produce hasta 1.000 gramos de eritropoyetina anuales, lo cual implica alrededor de 60 millones de dosis del medicamento de 2.000 unidades. Esto permite alcanzar un nivel de producción exportable que alcanza a toda Latinoamérica, Norte de África, Sudeste Asiático, Medio Oriente y Europa del Este. La alta calificación de ZELTEK la coloca en el grupo de las cuatro empresas más fuertes del sector junto a otras de China, India y Corea. Con estas capacidades, la firma genera 80 puestos de trabajo para personal altamente calificado.

Finalmente, ZOOVET (PRODUCTOS VETERINARIOS SA) se dedica a la fabricación de productos veterinarios, que cuentan con los más altos estándares de calidad en el mercado. Estos productos se comercializan a través de una red de vendedores con flota de vehículos propia: los distintos bienes que fabrica la firma se venden en Santa Fe, Corrientes, Córdoba, Provincia de Buenos Aires y Mendoza. A su vez, a lo largo del año pasado, han hecho las primeras incursiones en el mercado internacional, colocando parte de su producción en países limítrofes como Paraguay y estableciendo posibles bocas de expendio en Uruguay, Bolivia y diversos países de Centro América.

\section{AnÁlisis FODA: Fortalezas, Oportunidades, Debilidades Y AMENAZAS}

El enfoque FODA (Fortalezas, Oportunidades, Debilidades y Amenazas) es una herramienta clásica del gerenciamiento moderno y apunta a evaluar la situación estratégica de una organización, con el propósito de definir cursos de acción. Dentro del enfoque FODA, pueden reconocerse dos tipos de análisis.

En primer lugar, se encuentra el análisis interno de la entidad. Este diagnóstico se basa en conocer las fortalezas con las que cuenta la organización para alcanzar el logro de objetivos y simultáneamente, descubrir sus limitaciones, es decir las restricciones propias que no le permitan alcanzar metas de una manera efectiva.

En segundo lugar, se recurre a hacer un análisis de contexto. Dentro de esta perspectiva, se identifican las circunstancias ventajosas que pueden favorecer el 
crecimiento y desarrollo de la organización (oportunidades) y también aquellas tendencias del entorno que pueden perjudicarla, constituyéndose estas últimas en amenazas.

A continuación, se presenta el análisis FODA realizado para el Parque Científico Tecnológico Litoral Centro. El mismo se construyó a partir de entrevistas realizadas a las autoridades máximas de dicho parque, encuestas efectuadas a empresarios y documentos.

\section{FORTALEZAS}

\subsection{Existencia de un ambiente acorde para el desarrollo de actividades innovadoras}

El PTLC ha logrado conformar una atmósfera o clima de interacción entre empresarios, docentes e investigadores que promueve el surgimiento de actividades de innovación y facilita el desencadenamiento de nuevos negocios. El parque constituye un espacio organizado que integra la ciencia y la técnica, y la relaciona con el ámbito empresario, logrando que el conocimiento no solo se genere sino que también se transfiera. En este sentido, las empresas y emprendedores del parque han logrado aprovechar las externalidades positivas que surgen de la participación en un espacio de esta naturaleza, pudiendo incorporar estos elementos como parte de sus ventajas competitivas. Entre los espacios que promueven los procesos de interacción se destaca el comedor, ámbito que reúne y congrega a emprendedores e investigadores, facilitando el esparcimiento y generando momentos propicios para el surgimiento de ideas e intercambio de conocimientos (Cellino, 2012).

\subsection{Ubicación geográfica del parque}

Como se mencionó anteriormente, el PTLC está ubicado en una zona estratégica, integrando el corredor Coquimbo -Puerto Alegre, en el centro de las vías de comunicación terrestres que unen el MERCOSUR. Este posicionamiento estratégico hace que el parque participe de un gran circuito comercial, tanto local como regional. A su vez, este polo tecnológico se encuentra a 2 kilómetros del centro de la ciudad de Santa Fe, a 5 kilómetros del aeropuerto, y a $2 \mathrm{~km}$ de la autopista que lleva a la ciudad de Buenos Aires. En este sentido, su ubicación posibilita que empresarios e investigadores asociados a la institución tengan un rápido acceso a distintos centros, tanto financieros, educativos, como de consumo y recreación.

\subsection{Trayectoria y prestigio}

El PTLC constituye el primer polo tecnológico de Argentina. Viene funcionando en forma ininterrumpida desde el año 2002 y varias de las empresas que se han radicado allí han alcanzado reconocimiento internacional, por la calidad de los bienes fabricados y el nivel de los recursos humanos involucrados en sus procesos productivos. En este sentido, el parque se ha transformado en un referente en materia de transferencia tecnológica y constituye una marca registrada para todo futuro empresario que quiera iniciarse en el desarrollo de productos de base tecnológica. A su vez, el PTLC goza del apoyo de la Universidad Nacional del Litoral (UNL): una de las casas de estudio más destacadas de la República Argentina. Esta 
entidad es miembro fundador de la organización y varios de sus docentes e investigadores realizan actividades de asesoramiento y asistencia técnica dentro del parque. De acuerdo al criterio de indexación de publicaciones científicas SCOPUS, los trabajos científicos de la UNL son los más citados en el país. A su vez, en el Ranking Mundial de Universidades-desarrollado por el grupo de Investigación Español Scimago- la Universidad Nacional del Litoral ocupa el puesto $\mathrm{N}^{\circ} 874$, el más alto alcanzado por una casa de estudios de Argentina, exceptuando a la Universidad de Buenos Aires.

\subsection{Política de captación de recursos humanos}

El PTLC, la Universidad Nacional del Litoral y el CONICET SANTA FE vienen desarrollando una política conjunta de captación de recursos humanos altamente calificados, para potenciar la transferencia de tecnología y la actividad económica del parque. Esta política específica consiste en generar condiciones para la radicación de investigadores prestigiosos en el seno de la UNL y del CONICET, ofreciendo oportunidades para el crecimiento académico: esto incluye la posibilidad de dictar cursos de grado y postgrado, dirigir proyectos de investigación, formar becarios y tesistas. Como contra partida, se solicita al docente-investigador la prestación de servicios de consultoría técnica a spin off o empresas radicadas en el PTLC. A través de esta política, se logró la radicación de dos investigadores especializados en el área de biotecnología.

\section{DeBILIDADES}

\subsection{Falta de infraestructura}

El PTLC dispone de distintas limitaciones en materia de infraestructura que pueden condicionar su performance a futuro. En primer término, se puede reconocer como un factor a destacar las restricciones edilicias.

Hoy en día el polo necesita ampliar el área de incubación de empresas, que ha quedado chica en función de los requerimientos de ingreso al parque solicitados año a año7. Debido a la falta de presupuesto, las autoridades han tenido que optar entre ampliar la infraestructura destinada a satisfacer las necesidades de las empresas de biotecnología o desarrollar naves industriales para otras firmas. Esto derivó en que los emprendimientos del área de telecomunicaciones e informática no cuenten hoy en día con las instalaciones necesarias como para desarrollar adecuadamente sus actividades. A la falta de infraestructura física se suma la ausencia de rodados, que condicionan los desplazamientos del personal y de las autoridades hacia diversos destinos. De acuerdo a lo manifestado por los entrevistados, este último recurso es fundamental para el parque, ya que muchos de los fondos que consigue la organización se derivan de la actividad de lobby desarrollada ante diversas oficinas públicas (nacionales, provinciales y municipales) y para efectuar adecuadamente esta función es necesario contar con movilidad.

7De acuerdo a las estimaciones realizadas por las autoridades del parque, la incubadora de empresas debería contar hoy con $1200 \mathrm{~m}^{2}$ de construcción y sin embargo la edificación disponible no alcanza a la mitad. Resulta necesario ampliar el área de prerradicación, en el que existen tan sólo cuatro contenedores. 


\subsection{Insuficiente personal}

El PTLC no cuenta con el personal administrativo suficiente como para desarrollar adecuadamente sus actividades. A su vez, numerosas personas vinculadas al parque y que desarrollan funciones allí, no reciben ningún tipo de remuneración por sus acciones o los sueldos que perciben son abonados por el CONICET o en su defecto por la UNL. Dentro del primer grupo se encuentran todos los directores, el Gerente General y los miembros del Comité Ejecutivo. En este caso, el hecho de que la actividad que desarrollan sea ad honorem en muchos casos condiciona su nivel de participación y su involucramiento en los procesos decisorios respecto del futuro próximo del parque. Dentro del segundo, pueden reconocerse al personal de administración y al coordinador técnico (ambos registrados como personal de CONICET) y a la responsable del área contable, dependiente de la UNL. La situación laboral poco transparente de estos últimos, puede generar dificultades legales a futuro en caso de surgir imprevistos como accidentes, lesiones, desafectaciones o renuncias. En este sentido, sería recomendable que él mismo estuviese a cargo de la SAPEM PTLC.

\subsection{Falta de un presupuesto propio acorde}

Íntimamente relacionado con los dos puntos mencionados anteriormente, entre las principales restricciones mencionadas se encuentra la falta de presupuesto con el que cuenta la entidad. De acuerdo a lo manifestado por las autoridades del parque, del 100\% de los ingresos que obtiene la institución en concepto de cánones, patentamientos, etc. Solo un 30\% pasa a su propiedad, ya que el $70 \%$ restante queda en propiedad de CONICET y no siempre esta institución asociada decide reinvertir estos recursos en el parque (Cellino, 2012).

\section{OPORTUNIDADES}

\subsection{Crecimiento económico de la región central}

A partir de la expansión de la producción sojera, las Provincias de Córdoba, Santa Fe y Entre Ríos han experimentado un notable crecimiento económico. Motivo de esta expansión de la economía regional, las ganancias extraordinarias producidas por el sector agropecuario buscan alternativas de inversión en sectores no tradicionales que sean serias y confiables y que garanticen tasas razonables de rentabilidad en el mediano y largo plazo. EL PTLC junto con otros actores económicos, deberá desarrollar mecanismos financieros que permitan canalizar esos ahorros en proyectos productivos de base tecnológica, facilitando la ampliación de la capacidad tecno productiva de dicho espacio.

\subsection{Incremento del comercio entre países del MERCOSUR}

En esta última década, la República Federativa de Brasil se ha transformado en la sexta Economía Mundial, alcanzando un Producto Bruto Interno de 2.421.637 millones de dólares. A su vez, es el principal socio comercial de la Argentina, ya que le vende productos por un monto de USS 18.567.091.064. Como se sostuvo anteriormente, el PTLC se encuentra radicado en el centro de las comunicaciones 
terrestres del MERCOSUR y varios de los productos que fabrica encuentran un mercado potencial en expansión en este país vecino. A su vez, el ingreso de Brasil en los BRIC's amplía aún más las oportunidades de negocios de la firmas del Parque, en la medida que se facilitan los vínculos comerciales con países como Rusia o China, que pueden ser demandantes de productos de las empresas radicadas (principalmente de aquellas que fabrican productos asociados a la bio o nanotecnología aplicable en la producción agrícola o en el sector de la salud).

\subsection{Política de ciencia y tecnología que presenta el actual gobierno}

Durante la gestión de Cristina Fernández de Kirchner la política de ciencia y tecnología en Argentina ha sido jerarquizada, a través de la conformación de un ministerio y la ampliación de los recursos para el desarrollo de diversas actividades. A su vez, el sector público se ha transformado en un demandante de productos industriales argentinos con alto valor agregado y componente tecnológico, asegurando el "arrastre" que todo emprendimiento de esta naturaleza necesita para poder posicionarse en el mercado. Este comportamiento virtuoso del Estado Nacional también se ha expandido hacia otras esferas gubernamentales, como las Provincias y los Municipios. En este sentido, el PTLC ha sido favorecido por la demanda de productos médicos realizada por el Gobierno de Santa Fe, quien solicitó la fabricación de suero para atender la picadura de escorpión y medicamentos para el tratamiento del mal de Chagas. De este modo, el estado provincial colabora con el sostenimiento de la actividad tecnológica desarrollada en el polo. Hasta hoy en día, la política de ciencia y tecnología inaugurada durante la gestión kirchnerista, continúa a grandes rasgos en la presidencia del ingeniero Mauricio Macri y se espera que se transforme en una política de estado para la República Argentina.

\subsection{Convenios con empresas multinacionales en las áreas de biotecnología}

El desarrollo de productos con alto contenido tecnológico en el área de biotecnología requiere de grandes inversiones y simultáneamente implica tener desarrollados canales de comercialización muy específicos para poder colocar la producción. La mayor parte de las empresas radicadas hoy en el parque no cuentan con estas dos herramientas claves para desarrollar su negocio y consideran que a través de acuerdos con empresas multinacionales podrán conseguir ambos objetivos: capitales para invertir y una demanda cautiva para sus bienes o servicios ${ }^{8}$. Actualmente existe diálogo con diversas empresas extranjeras que están analizando la posibilidad de establecer convenios específicos con el parque y con firmas radicadas en él. Por supuesto, estos convenios deben ser firmados tratando de mantenerse en un plano de igualdad con las firmas multinacionales, para tratar de evitar acuerdos leoninos que perjudiquen los intereses del parque o de las empresas locales.

8 Desde el año 2002 al 2011 fueron presentadas en la UNL 40 patentes en el área de biotecnología correspondiente a distintas empresas. Muchas de ellas todavía no han podido transformarse en negocios por falta de financiamiento 


\section{AMENAZAS}

\subsection{Dificultades con la conectividad de la localidad}

Si bien la ciudad de Santa Fe es una de las localidades del país que mejor infraestructura física posee, debido a su importante red eléctrica, de agua, gas y servicios cloacales, junto con su amplio espectro de calles y avenidas asfaltadas es menester destacar que presenta algunas deficiencias en materia de comunicación que fueron destacadas por los entrevistados. En este sentido, las personas consultadas sostuvieron que el aeropuerto de Arroyo Corto en Santa Fe no cuenta con la frecuencia de vuelos de cabotaje necesarios como para que emprendimientos como el PTLC puedan desarrollarse adecuadamente. Es necesario recordar que este tipo de iniciativas convoca un afluente significativo de hombres de negocios y funcionarios públicos, que deben contar con una frecuencia diaria de vuelos que les permitan transitar sin demoras la distancia entre la ciudad de Buenos Aires y la cabecera provincial. Hoy en día, además de la ausencia de frecuencias, se registran innumerables demoras y deficiencias en la prestación del servicio, que llevan a que muchos funcionarios y empresarios que visitan el parque termine optando por trasladarse en ómnibus, con la consecuente pérdida de tiempo y confort que esta medida implica. Desde el año 2011, existe un compromiso del Estado Nacional de transformar la aerostación de Arroyo Corto en Aeropuerto Internacional, pero hasta la fecha no se han recibido los fondos necesarios para ampliar las pistas y desarrollar el balizamiento, requisitos fundamentales para que este cambio de categoría pueda concretarse.

\subsection{Creación excesiva de parques}

En los últimos años, a raíz del auge que existe respecto a la importancia de la innovación como motor del desarrollo económico, han surgido en el país numerosos parques y polos tecnológicos, algunos de los cuales carecen de los requisitos necesarios como para ser considerados entes de esta naturaleza (Cellino, 2012). La excesiva proliferación de parques de baja actividad y con bajo impacto económico y social puede ser perjudicial para un ente como el PTLC, que se encuentra en proceso de crecimiento y consolidación. En términos generales, podrían existir al menos dos efectos negativos que afecten el funcionamiento del parque: en primer lugar, que el estado decida disminuir la inversión pública en estos espacios por la falta de resultados palpables e identificables en materia de innovación tecnológica. En segundo lugar, que se vea disminuida la inversión privada, por la desconfianza del sector respecto a la performance de estos espacios.

\subsection{Marco institucional débil para el desenvolvimiento de la actividad}

Argentina no tiene una trayectoria en el impulso de actividades de ciencia y tecnología que buscan comercializarse en el mercado, esto ha derivado en la existencia de importantes vacíos legales, que complotan contra el normal desenvolvimiento de la actividad. Actualmente se han desarrollado importantes avances respecto a la legislación sobre patentamiento y propiedad intelectual pero 
no existe aún un marco legal acorde en materia de distribución de regalías, es decir, la distribución de las ganancias que surgen a partir de la comercialización de productos basados en investigación y desarrollo, que se materializan a través de actividades de cooperación entre actores públicos y privados.

\subsection{Posible emigración de científicos}

En Argentina, si bien los salarios que perciben los docentes universitarios e investigadores científicos son superiores a la media nacional, se encuentran rezagados en relación a lo que percibe una persona de la misma calificación por igual tarea en los países desarrollados o incluso en algunas naciones vecinas (medido en dólares). Si bien en los últimos años, el estado nacional jerarquizó la actividad docente universitaria y la investigación mejorando los salarios, lo cual generó una disminución en la fuga de cerebros, hoy en día el proceso inflacionario esta licuando las remuneraciones del sector. Claramente el PTLC resulta perjudicado por esta política, ya que el capital humano y el conocimiento son un insumo para el desarrollo de sus actividades. Hasta la fecha, el ente no ha sido perjudicado por esta cuestión, pero no descartan la posibilidad de que a futuro, algún investigador de su cuerpo permanente de prestadores de servicios emigre debido a un mejor sueldo o mejores condicionales laborales en otro país.

\subsection{Problemas históricos en la conexión entre empresarios y hombres de ciencia}

En Argentina existe un problema histórico de desconexión entre la ciencia y la actividad empresarial. De acuerdo a Azpiazu y Bramuglia (1992), esto de se debe a diferencias en el comportamiento de ambos actores y a la existencia de distintas formas de trabajo, que son difíciles de compatibilizar. Respecto a las cuestiones de comportamiento, en primer término, se observa que los empresarios y los hombres de ciencia manejan tiempos diferentes: el empresario necesita soluciones inmediatas a los problemas tecnológicos, productivos y organizacionales de su firma mientras que, en la esfera de la ciencia, las soluciones suelen alcanzarse luego de procesos de maduración. En segundo lugar, el empresario tiene como objetivo fundamental el lucro y la obtención de beneficios, mientras que en el ámbito de la ciencia el lucro está en un segundo plano y lo primordial es la obtención de reconocimiento y prestigio de carácter corporativo. En tercer término, en la esfera empresarial el status de empresario exitoso se alcanza en función de la cantidad y calidad de las empresas generadas, mientras que, en la academia el status es función de la cantidad de publicaciones científicas efectuadas. En cuanto a las formas de trabajo, en la esfera empresarial predomina el trabajo por objetivos y la fijación de metas a cumplir en lapsos cortos de tiempo en un contexto de trabajo regulado mientras que, en la esfera científica, prima la libertad en la elección de los proyectos y los horarios laborales flexibles.

En el PTLC las autoridades realizan tareas de sensibilización para que ambos actores sean conscientes de estas diferentes metodologías de abordaje de los problemas y respeten los tiempos y la idiosincrasia de sus interlocutores. Sin embargo, en numerosas ocasiones los vínculos entre empresarios e investigadores igualmente se desgastan, aunque nunca llevaron a que fracase un proyecto. 


\section{CONCLUSIONES}

Como se sostuvo en la introducción del trabajo, en el contexto actual de la globalización se están generando cambios tecnológicos que alteran el funcionamiento del sistema capitalista. Esencialmente, se observa a escala mundial la existencia de una nueva forma de producir bienes y servicios, que tiene como factor clave al conocimiento. Si bien los saberes han sido siempre un insumo de la economía, en los últimos años se transformaron en un elemento fundamental de los procesos tecno-productivos.

Las grandes economías captaron que debían realizar un cambio en la forma de fabricar bienes y servicios, ya que el factor mencionado constituía un limitante para el crecimiento y la acumulación de capital. Debido a esta cuestión, apostaron al desarrollo de nuevos sectores industriales intensivos en conocimientos tecnológicos y científicos: la industria del software, la biotecnología, la microelectrónica, entre otros.

A partir de esta premisa, se diseñaron numerosos mecanismos de política que buscan profundizar u optimizar el uso de este recurso. Los parques tecnológicos, constituyen uno de los instrumentos más utilizados para alcanzar dicho objetivo. De acuerdo a Rodríguez Pose (2012), la mayoría de los académicos reconoce a los PCT como una iniciativa - generalmente pública- de creación de un área geográfica delimitada y destinada a favorecer el desarrollo y la aplicación de actividades científicas y tecnológicas. Esta área tiene como fin promover y albergar instituciones de investigación y empresas intensivas en el uso y la generación de conocimiento, entre las que se estimula la producción y la transferencia.

El origen de los mismos se encuentra en América del Norte, habiéndose extendido su aplicación a Europa y posteriormente al sudeste de Asia y América Latina. En términos generales, se observa a nivel mundial una importante heterogeneidad respecto a su forma organizativa, los servicios que los mismos prestan y los sectores que se buscan potenciar. Es debido a esto que resulta difícil construir un modelo o tipología de parque que sea capaz de resumir las diferentes experiencias internacionales.

En Argentina, las primeras iniciativas de esta naturaleza datan de la década de 1990, existiendo actualmente cinco parques tecnológicos operativos, uno en proceso de implantación y uno en carácter de proyecto, siendo el Parque Científico Tecnológico del Litoral Centro (PTLC) el que ha alcanzado mayor desarrollo. Este parque se encuentra radicado en la ciudad de Santa Fe y tiene como principal objetivo la interacción entre el sector científico y empresarial para lograr como fin último la transferencia de tecnología a la sociedad. A la fecha, el PTLC constituye la iniciativa de esta naturaleza más exitosa del país, habiendo generado más de 300 puestos de trabajo en forma directa y exportado 42 millones de dólares.

Mediante una serie de entrevistas a autoridades y empresarios ligados a esta organización, se logró construir un análisis de las fortalezas, debilidades, oportunidades y amenazas que la misma presenta para su desempeño futuro, con el propósito de entender el papel que tiene como instrumento de política pública.

En primer lugar, se reconoció como un atributo importante de éxito su trayectoria y prestigio. El PTLC es considerado el primer polo tecnológico del país y funciona en forma ininterrumpida desde el año 2002, existiendo varias firmas 
exitosas que se han radicado allí, alcanzando reconocimiento internacional. Esto se debe a la calidad de los bienes fabricados y al nivel de los recursos humanos involucrados en los procesos productivos, que alcanzan los mejores estándares del mercado.

Además el PTLC presenta la particularidad de encontrarse situado en una zona estratégica, integrando el corredor Coquimbo-Porto Alegre, en el centro de las vías de comunicación terrestres que unen el MERCOSUR. Este posicionamiento hace que el parque pueda ser participe de los flujos económicos y comerciales ligados a este mercado común.

En tercer término, como una importante fortaleza las autoridades reconocieron estar desarrollando una intensa política de captación de recursos humanos para potenciar la transferencia de conocimiento y tecnología. Esta política consiste en otorgar ventajas diferenciales a investigadores nacionales y extranjeros para que se radiquen en Santa Fe y colaboren con las acciones que lleva a cabo la organización.

Otra característica favorable del PTLC es haber conformado un ambiente acorde para el desarrollo de actividades innovadoras. Se debe reconocer que ha logrado desarrollar una atmósfera o clima de interacción entre empresarios, docentes e investigadores que promueve el surgimiento de emprendimientos.

Por su parte, las externalidades positivas de su entorno, constituyen una fuente de crecimiento económico para la región central del país y pueden ser un elemento favorable para la consolidación del PTLC. En este sentido, el PTLC dispone de ventajas competitivas capaces de atraer las ganancias extraordinarias que genera hoy día el sector agropecuario. En su seno existen empresas especializadas en conocimiento que son capaces de asegurar altos niveles de rentabilidad en el mediano y largo plazo, superiores a las que se obtien en en cualquier otro tipo de actividad.

EL PTLC junto con otros actores económicos, deberá desarrollar mecanismos financieros que permitan canalizar esos ahorros en proyectos de base tecnológica, facilitando la ampliación de la capacidad tecno productiva de dicho espacio.

A su vez, existe un incremento del comercio entre países del MERCOSUR, y el polo presenta en este contexto una interesante oportunidad de expansión. Hoy en día varios de los productos que se fabrican en el parque cuentan con un mercado potencial en Brasil, situación que debe ser aprovechada por los actores que integran el mismo con el propósito de poder consolidar la iniciativa.

Por otro lado, entre las oportunidades detectadas por los entrevistados se remarcó la presencia de políticas activas en materia de Ciencia y Tecnología impulsadas por el gobierno nacional, que pueden ser usufructuadas para mejorar la performance general del polo. En los últimos años el sector público se ha transformado en un demandante de productos tecnológicos, asegurando la demanda cautiva que todo emprendimiento de esta naturaleza necesita para poder posicionarse en el mercado.

Simultáneamente, la expansión del parque requiere el establecimiento de convenios con empresas transnacionales para desarrollar canales de comercialización y obtener inversiones, lo cual implica potenciar acuerdos de cooperación público-privada, situación que fue mencionada explícitamente por las personas consultadas. 
Sin embargo, más allá de los atributos del parque y de la presencia de elementos favorables en el entorno, se debe aclarar que también existen diversas cuestiones que pueden condicionar su desempeño futuro, y por lo tanto su incidencia como instrumento de política pública. Entre ellas puede reconocerse cuestiones relativas a la falta de financiamiento acorde, la limitada infraestructura, la insuficiencia de personal administrativo y la presencia de directores trabajando adhonorem. Estas cuestiones parecen de alguna forma contrapesar muchos de los elementos positivos destacados en párrafos anteriores y requieren ser atendidas en breve por ser de carácter estructural.

Además, existen varias amenazas del entorno que las autoridades del PTLC deben considerar, si buscan que su organización alcance un futuro próspero. En relación a esta cuestión, resulta menester mencionar el importante aumento de parques científicos y tecnológicos que se ha producido en Argentina en los últimos años. Muchos de estos nuevos parques no constituyen ámbitos reales para el desarrollo de la ciencia y la tecnología, sino que son más bien cascaras vacías para la conformación de negocios privados poco transparentes con recursos públicos. En este sentido, el PTLC deberá ser capaz de diferenciarse de estos espacios para poder captar inversiones reales que fortalezcan su desempeño y su imagen institucional.

Otro punto a considerar es que la Argentina no tiene una trayectoria en el impulso de actividades de ciencia y técnica que busquen comercializarse en el mercado, esto ha derivado en la existencia de importantes vacíos legales, que complotan contra el normal desenvolvimiento de la actividad. Para contrarrestar este fenómeno, las autoridades del PTLC deberán sensibilizar a funcionarios públicos, diputados y senadores para que trabajen en el diseño de un cuerpo legal acorde a las necesidades de las empresas de base tecnológica.

También debe reconocerse como una cuestión importante a trabajar en materia de política institucional, la posible fuga de cerebros en el sistema científico tecnológico, a raíz del deterioro del salario real que registran los investigadores y científicos argentinos. La imposibilidad de pagar salarios similares a los percibidos en los países desarrollados genera un drenaje de recursos humanos que afecta los resultados de la ciencia y la tecnología nacional y puede impactar en un futuro inmediato en la performance del parque.

Aquí nuevamente resultará crucial la tarea de alertar a los dirigentes políticos $\mathrm{y}$ funcionarios sobre esta cuestión y conformar consensos u alianzas dentro del sistema científico tecnológico, para elevar propuestas al ejecutivo nacional que morigeren esta cuestión.

Finalmente, resulta necesario recordar que en Argentina existe un problema histórico de desconexión entre la ciencia y la actividad empresarial, que complota contra la transferencia de tecnología y la conformación de espacios como el PTLC. Esto se debe a diferencias en el comportamiento de ambos actores y a la existencia de distintas formas de trabajo, que son difíciles de compatibilizar. Para trabajar este punto es prioritario la organización de espacios de reflexión conjunta entre ambos actores. Esta actividad puede reforzarse contratando personal especializado en las tareas de enlace y articulación de ambas esferas de actuación, capaces de compatibilizar dos mundos que presentan códigos y lenguajes diferentes.

De este modo, puede decirse que el desenlace respecto al futuro del PTLC como instrumento de política pública presenta un final abierto, que dependerá de la 
capacidad de sus autoridades para aprovechar oportunidades y desarticular amenazas, utilizando recursos propios y disimulando falencias.

\section{BIBLIOGRAFÍA}

Adán, C. (2012). El ABC de los parques científicos. Seminarios de la Fundación Española de Reumatología, Vol. 13, n ${ }^{\circ}$ 3, pp. 85-94.

Azpiazu, D., Bramuglia, C. (1992). Indicadores económicos de las actividades científico-tecnológicas: una comparación internacional en E. Oteiza (comp.) La política de investigación científica y tecnológica argentina. Historia y perspectivas, Buenos Aires, Centro Editor de América Latina, pp. 32-59.

BENKO, G. (1998). El impacto de los tecnopolos en el desarrollo regional: Una revisión crítica. Revista Latinoamericana de Estudios Urbano RegionalesEURE, Vol. 24, $\mathrm{n}^{\mathrm{O}} 73$.

Barbieri, J. C (1994). Polos tecnológicos e de modernización, notas sobre la experiencia brasileira. Revista de Administración de Empresas, Vol. 34, $\mathrm{N}^{\circ}$ 5, pp. 21-31.

Botella, C.; Suárez, I. (2012). Innovación para el desarrollo en América Latina una aproximación desde la cooperación internacional, Madrid, Fundación Carolina/CeALCI.

Cellino, A (2011). Dificultades para la formación de empresas de base tecnológica. Conferencia dictada en el worshop de Política Tecnológica del Ministerio de Ciencia y Tecnología (MINCyT): estudios de caso y experiencia en Argentina. Buenos Aires, 5, 6 y 7 de Octubre.

Cellino, A. (2012). Parques Científicos - Tecnológicos y el desarrollo regional. Ámbitos para la innovación y la inversión. Conferencia dictada en el auditorio principal UAT CONICET Bahía Blanca. Bahía Blanca, 18 de Abril.

Chicoye, C. (1989). Análisis del fenómeno de parques científicos en Francia, Santiago de Chile, ILPES/CEPAL-Universidad Católica de Chile.

Etzkowitz, H. y Leydesdorff, L. (1997). Triple Helix of University-IndustryGovernment relations. En H.Etkowitz, H. y LLeydesdorff (eds):Universities and the global Knowledge Economy. A triple Helix of University-IndustryGovernment relations. London, printer publishers, pp. 45-67.

Hoffmann, M.; Mais, I; Amal, M. (2010). Planejamento e gestao de parques científicos e tecnológicos: um aanálise comparativa. Economia Global e Gestao, Vol. 15, N 10, pp. 89-107.

Koh, F.; Koh, W.; Tschang, F. (2005). An analytical framework for science parks and technology districts with an application to Singapore. Journal of Business Venturing, $\mathrm{N}^{\circ}$ 2O, pp. 217-239.

Massey, D.; Quintas, P.; Wield, D. (1992). High Tech Fantasies, London, Routledge.

Malezieux, J. (1993). Hautes technologies- noveauxespacesd'activitésdeveloppement local et regional-apparences et realités. Réflexions sur le aménagementsfracais. En Actas del V Collaque Internacional Novas Tecnologías e Desenvolvimento Regional,Portugal, Universidad de Coimbra, Pp-157-167.

Lahorgue, M.A. (2010). Sistema de indicadores de innovación: el caso de las incubadoras de empresas en Brasil, en VIII Congreso Ibero-Americano de Indicadores de Ciencia y Tecnología,Madrid, 5, 6 y 7 de Septiembre. 
Ondategui Rubio, J. (2001). Parques Científicos y Tecnológicos: los nuevos espacios productivos del futuro. Investigaciones Geográficas (Esp), Universidad de Alicante, España, $\mathrm{N}^{\circ}$ 25, pp 95-118.

Park, S. H. (2001). Globalisation and local innovation system: the implementation of government policies to the formation of Science Parks in Japan”, Al \& Society, $\mathrm{N}^{\mathrm{o}} 15$, pp. 263-279.

Perez, C. (1996). Nueva concepción de la tecnología y sistema nacional de innovación. Cuadernos del Centro de Estudios del Desarrollo-CENDES, Año $13, \mathrm{~N}^{\circ} 31, \mathrm{pp} .9-33$.

Rodríguez Pose, A. (2012). Los parques científicos y tecnológicos en América Latina. Un análisis de la situación actual, New York, BID.

UNIVERSIDAD NACIONAL DEL LITORAL (2012a). Comunicación para la construcción y el fortalecimiento de vínculos: estrategias de comunicación para la vinculación tecnológica de la UNL en Gestión de la Comunicación: Aportes y Desafíos de la Vinculación Tecnológica, Santa Fe, Ediciones UNL, 2012.

UNIVERSIDAD NACIONAL DEL LITORAL (2012b). Gabinete para emprendedores: un espacio de incubación cercano a los estudiantes y docentes para promover emprendimientos en Gestión de la Comunicación: Aportes y Desafíos de la Vinculación Tecnológica, Santa Fe, Ediciones UNL, 2012.

Saxenian, A. (1990). Regional Network and the resurgence or Sillicon Valley. California Management Review, $\mathrm{N}^{\circ} 33$, pp.89-112.

Saxenian, A. (1991). The origins and dinamics of production networks in Silicon Valley. Research Policy, $\mathrm{N}^{\circ}$ 20, pp. 423-437.

Shearmour, R. y Doloreaux, D. (2000). Science parks: actors or reactors Canadian science parks in their urban context, Environment and Planning, Vol. 32, pp. 1065-1082.

SILICON VALLEY INSTITUTE FOR REGIONAL STUDIES (2013). Silicon Valley Index: people, economy, society, place, governance, Mountain View, Silicon Valley Community Foundation.

SILICON VALLEY INSTITUTE FOR REGIONAL STUDIES (2014). Silicon Valley Index: people, economy, society, place, governance, Mountain View, Silicon Valley Community Foundation.

\section{ENTREVISTAS}

Entrevista efectuada al Dr. Amadeo Cellino, Gerente General del Parque Científico Tecnológico Litoral Centro, realizada por el Dr. José Ignacio Diez. 18 de Abril de 2017. 